\title{
Numerical Solution of the Minimal Surface Equation*
}

\author{
By Paul Concus
}

1. Introduction. A method for the numerical solution of the two-dimensional nonlinear magnetostatic-field equation has been studied recently by the author [1]. It is based on the iterative solution, by nonlinear successive overrelaxation, of a set of nonlinear difference equations approximating the original quasi-linear, elliptic, partial differential equation. The details of the method, its relationship to other methods, and its effectiveness in solving a magnetostatic test problem are described in [1]. The purpose here is to test the method on the minimal surface equation, which is of the same form but is, in general, more nonlinear and difficult to solve. A slight change in the method is useful for this equation in order to reduce the number of computational operations required per iteration. The numerical results obtained for the test problems are favorable.

2. The Problem. The minimal surface equation is the Euler equation for Plateau's problem in restricted, or nonparametric, form, which can be stated as follows [3, $\$ 18.9]$ : Let $f(x, y)$, a single-valued function defined on the boundary $C$ of a simply connected region $R$ in the $x-y$ plane, represent the height of a given space curve $\Gamma$ above the point $(x, y)$ on $C$. Let $u(x, y)$ represent the (single-valued) height, above the point $(x, y)$ in $R$, of the surface of minimal area through $\Gamma$. Then the problem, in variational form, is that of finding a function $u(x, y)$ twice continuously differentiable in $R$ satisfying

$$
u(x, y)=f(x, y) \quad \text { on } \quad C
$$

and minimizing the surface area

$$
A=\iint_{R}\left(1+u_{x}^{2}+u_{y}^{2}\right)^{1 / 2} d x d y
$$

The Euler equation corresponding to Eq. (2) in vector operator notation is

$$
\boldsymbol{\nabla} \cdot\left[\gamma\left(|\nabla u|^{2}\right) \nabla u\right]=0
$$

where

$$
\gamma\left(|\boldsymbol{\nabla} u|^{2}\right)=\left(1+|\boldsymbol{\nabla} u|^{2}\right)^{-1 / 2} .
$$

If the differentiations in Eq. (3) are carried out and the equation multiplied by $\left(1+|\nabla u|^{2}\right)^{3 / 2}$, one obtains the more familiar form of the minimal surface equation,

$$
\left(1+u_{y}^{2}\right) u_{x x}-2 u_{x} u_{y} u_{x y}+\left(1+u_{x}^{2}\right) u_{y v}=0,
$$

Received July 22, 1966. Revised November 14, 1966.

* This work done under the auspices of the U.S. Atomic Energy Commission. 
from which the ellipticity and quasi-linearity may be directly observed. In order to obtain symmetric difference equations, however, it is better to work either from Eq. (3) or from the variational integral Eq. (2).

The principal family of test problems considered is of the same form as that studied in [1]. Here it is to find the minimal surface through the curve $\Gamma$,

$$
\begin{aligned}
& x=0, z=0 \\
& x=2, z=0, \quad 0 \leqslant y \leqslant 1, \\
& y=1, z=0 \\
& y=0, z=K \sin (\pi x / 2), \quad 0 \leqslant x \leqslant 2,
\end{aligned}
$$

for several values of $K$. When the symmetry about $x=1$ is used, the problem reduces to solving Eq. (3) in the region $0<x<1,0<y<1$ with

$$
\begin{aligned}
& u=0 \text { on } x=0 \text { and } y=1, \\
& u=K \sin (\pi x / 2) \text { on } y=0,
\end{aligned}
$$

and

$$
\partial u / \partial x=0 \text { on } x=1 .
$$

The test problem studied in [2] of solving Eq. (3) in the region $0<x<1,0<y$ $<1$ with boundary conditions

$$
u=\left[\cosh ^{2} y-x^{2}\right]^{1 / 2}
$$

on the perimeter is also briefly considered.

3. The Method. The method of [1] can be applied directly to the present problem. As in Eq. (14) of [1], for a square mesh of width $h=1 / N$ ( $N$ an integer), one is led to the following nonlinear difference equation for Eq. (3) at an interior point:

$$
\begin{array}{r}
f_{i j}=\gamma_{\bar{i} \bar{j}}\left(2 u_{i j}-u_{i-1, j}-u_{i, j-1}\right)+\gamma_{\bar{i}+1, \bar{j}}\left(2 u_{i j}-u_{i+1, j}-u_{i, j-1}\right) \\
+\gamma_{\bar{i}, \bar{j}+1}\left(2 u_{i j}-u_{i-1, j}-u_{i, j+1}\right)+\gamma_{\bar{i}+1, \bar{j}+1}\left(2 u_{i j}-u_{i+1, j}-u_{i, j+1}\right)=0, \\
(1 \leqslant i \leqslant N-1,1 \leqslant j \leqslant N-1) ;
\end{array}
$$

here $\gamma_{\bar{i} \bar{j}}=\gamma\left(|\nabla u|^{\frac{2}{i}}\right)$ denotes $\gamma$ for the mesh cell with center $(i-1 / 2, j-1 / 2)$, which is evaluated by use of

$$
\begin{aligned}
|\boldsymbol{\nabla} u|_{i j}^{2}= & \frac{1}{2 h^{2}}\left[\left(u_{i j}-u_{i-1, j}\right)^{2}+\left(u_{i j}-u_{i, j-1}\right)^{2}\right. \\
& \left.+\left(u_{i, j-1}-u_{i-1, j-1}\right)^{2}+\left(u_{i-1, j}-u_{i-1, j-1}\right)^{2}\right] .
\end{aligned}
$$

Eq. (7) approximates Eq. (3) locally to $O\left(h^{2}\right)$, and Eq. (8) approximates $|\nabla u|^{2}$ at the center of the cell to $O\left(h^{2}\right)$. Eq. (7) may be derived directly from either the variational integral, Eq. (2), or the differential equation, Eq. (3), as long as Eq. (8) is used to approximate $|\nabla u|^{2}$. Along the symmetry boundary, $i=N$, in the first family of test problems, one has 


$$
\begin{aligned}
f_{N j}= & \gamma \bar{N}_{\bar{j}}\left(2 u_{N j}-u_{N-1, j}-u_{N, j-1}\right) \\
& +\gamma_{\bar{N}, j+1}\left(2 u_{N j}-u_{N-1, j}-u_{N, j+1}\right)=0 \quad(1 \leqslant j \leqslant N-1) .
\end{aligned}
$$

The difference equations are solved iteratively by computing $u_{i j}^{k+1}$, the $(k+1)$ th approximation to $u_{i j}$, from

$$
u_{i j}^{k+1}=u_{i j}^{k}-\omega \frac{f_{i j}\left[u_{11}^{k+1}, \cdots, u_{i-1, j}^{k+1}, u_{i j}^{k}, \cdots, u_{N, N-1}^{k}\right]}{\frac{\partial f_{i j}}{\partial u_{i j}}\left[u_{11}^{k+1}, \cdots, u_{i-1, j}^{k+1}, u_{i j}^{k}, \cdots, u_{N, N-1}^{k}\right]},
$$

where $\omega$ is the relaxation parameter. For the test problems, the iteration is ordered by letting $i$ increase through all its values for each successively larger value of $j$. Only the case in which $\omega$ does not vary from mesh point to mesh point is considered.

The finite-difference equations thus obtained have a symmetric Jacobian matrix that is block tridiagonal, each block of which is itself tridiagonal. Although the Jacobian matrix is positive-definite, it does not, in general, have diagonal dominance nor does it have off-diagonal elements all of the same sign and opposite to that of the diagonal elements. If the differential equation, Eq. (3), were linear-that is, if $\gamma$ were a function of $x$ and $y$ alone-then the above difference equations would reduce to the usual five-point ones and the iterative scheme, Eq. (9), would reduce to point-successive overrelaxation with a coefficient matrix having property (A).

The estimation of the optimal value of the relaxation parameter, $\omega$, is important in order to obtain the most rapid convergence of the iteration. For the magnetostatic test problem [1,4] (and also for a mildly nonlinear equation [5]), it was observed that the optimal relaxation parameter is essentially equal to its asymptotically optimal value (the limiting parameter that yields fastest convergence in a small neighborhood of the solution). This asymptotic parameter, furthermore, is essentially equal to that estimated by the formulas valid for estimating the optimal parameter for linear point successive overrelaxation applied to a matrix possessing property (A), even though the Jacobian matrix does not, in general, have this property. The usefulness of this feature is discussed further when the results for the test problems are presented.

Another item remaining to be discussed concerns the device to speed the computations for solving the minimal surface equation. Note that Eq. (9) requires the evaluation of $|\nabla u|^{2}, \gamma\left(|\nabla u|^{2}\right)$, and $d \gamma\left(|\nabla u|^{2}\right) / d|\nabla u|^{2}$ for the surrounding mesh cells each time a value of $u$ is changed at a mesh point. The repeated calculation of $\gamma$ from Eq. (4) is undesirable, because it involves the time-consuming operation of extracting a square root. If, however, the previously calculated value of $\gamma$ is available, then one can save time by instead performing only one Newton iteration,

$$
\gamma_{n+1}=1 / 2\left[\gamma_{n}+\left(1+|\nabla u|_{n+1}^{2}\right) / \gamma_{n}\right] .
$$

to approximate the new value, $\gamma_{n+1}$, by use of the old value, $\gamma_{n}$, and the newly calculated $|\nabla u|_{n+1}^{2}$. The error introduced by using Eq. (10) instead of Eq. (4) is in keeping with the other local truncation errors of the method. The derivative can, of course, be computed from $\gamma$ and $|\nabla u|^{2}$ by use of the relationship

$$
d \gamma\left(|\boldsymbol{\nabla} u|^{2}\right) / d|\boldsymbol{\nabla} u|^{2}=-\gamma /\left[2\left(1+|\boldsymbol{\nabla} u|^{2}\right)\right] .
$$




\section{Test Results.}

4.1. Condition number. Before discussing the numerical results, it is helpful to introduce a quantity with which to compare the degrees of nonlinearity for the different values of $K$ in the first family of test problems. Such a quantity is the condition number of the coefficient matrix for Eq. (3),

$$
\left(\begin{array}{ll}
\gamma+2 \gamma^{\prime} u_{x}^{2} & 2 \gamma^{\prime} u_{x} u_{y} \\
2 \gamma^{\prime} u_{x} u_{y} & \gamma+2 \gamma^{\prime} u_{y}^{2}
\end{array}\right) .
$$

For a given $u_{x}, u_{y}, x$, and $y$, it has eigenvalues $\gamma$ and $\gamma+2\left(u_{x}{ }^{2}+u_{y}{ }^{2}\right) \gamma^{\prime}$, the product of which must be positive for the problem to be elliptic. For the magnetostatic test problem of [1] with $\gamma=\left(10^{-4}+|\nabla u|^{2}\right) /\left(1+|\nabla u|^{2}\right)$, both eigenvalues are uniformly greater than or equal to $10^{-4}$ for all $|\nabla u|^{2}$. For the minimal-surface equation, the

$(1,1)$
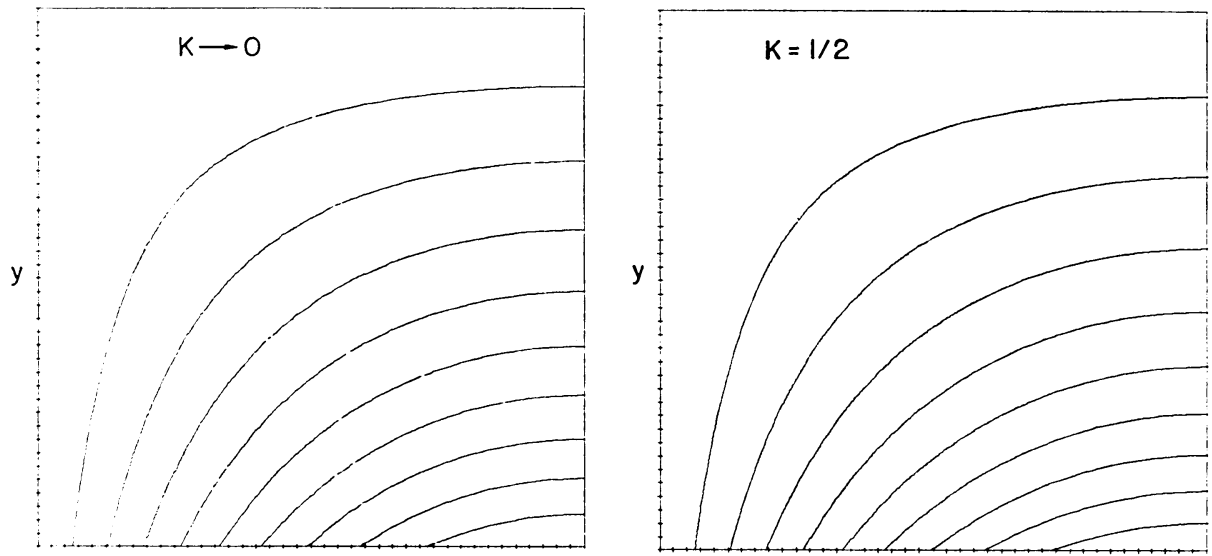

$(0,0)$

$\mathrm{x}$

$(0,0)$

$x$

$(1,1)$ $(1,1)$
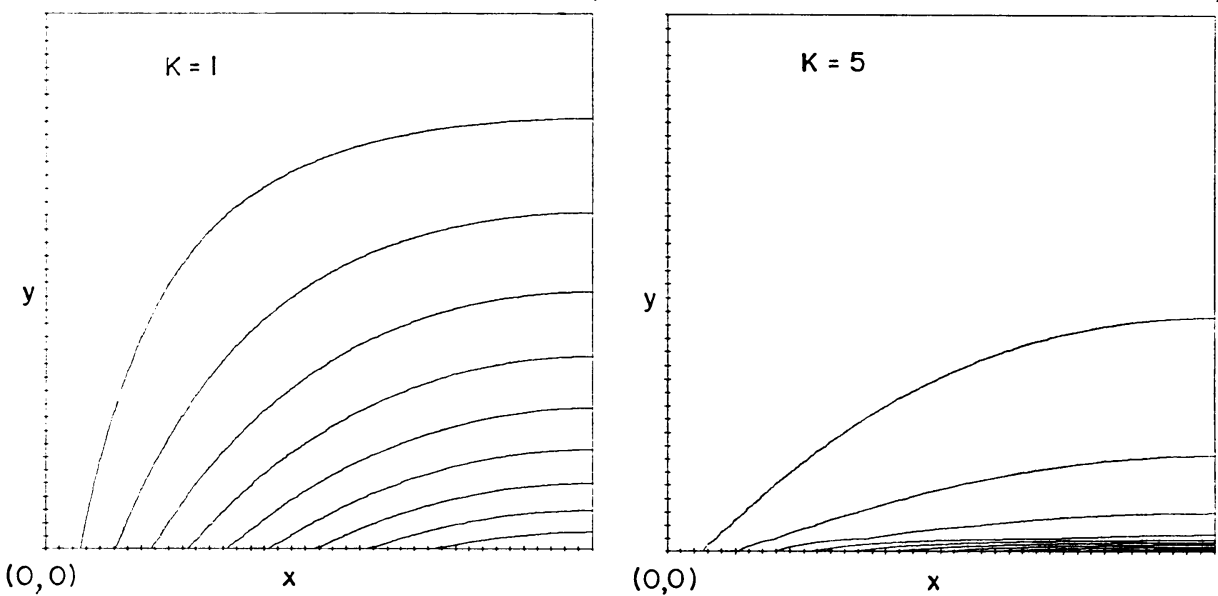

Figure 1. Test problems solutions. Contour lines are drawn every $K / 10$. 
eigenvalues are not uniformly bounded away from zero, but are merely greater than or equal to $\left(1+|\nabla u|_{M}^{2}\right)^{-3 / 2}$, where $|\nabla u|_{M}^{2}$ is the least upper bound for $|\nabla u|^{2}$.

Define the condition number to be the least upper bound of the ratio of the larger to the smaller eigenvalue of the coefficient matrix, when $u$ is the solution of the test problem. For a linear problem, this number is 1. For the magnetostatic test problem, this number could be at most 2 . For the Plateau test problem, it is $\left(1+|\nabla u|_{M}^{2}\right)$ and, hence, can be made as large as desired by choosing appropriate boundary values for $u$. In general, as $K$ becomes larger, the test problem is more nonlinear, the condition number and spectral radius of the asymptotic iteration matrix are larger, and the convergence rate is slower.

4.2. Results for first problem. The results for the first family of test problems Eq. (5), are given in Figs. 1 and 2 and Tables I, II, and III. Three values of $K$ are used-1/2, 1, and 5-which correspond to increasing degrees of nonlinearity. The

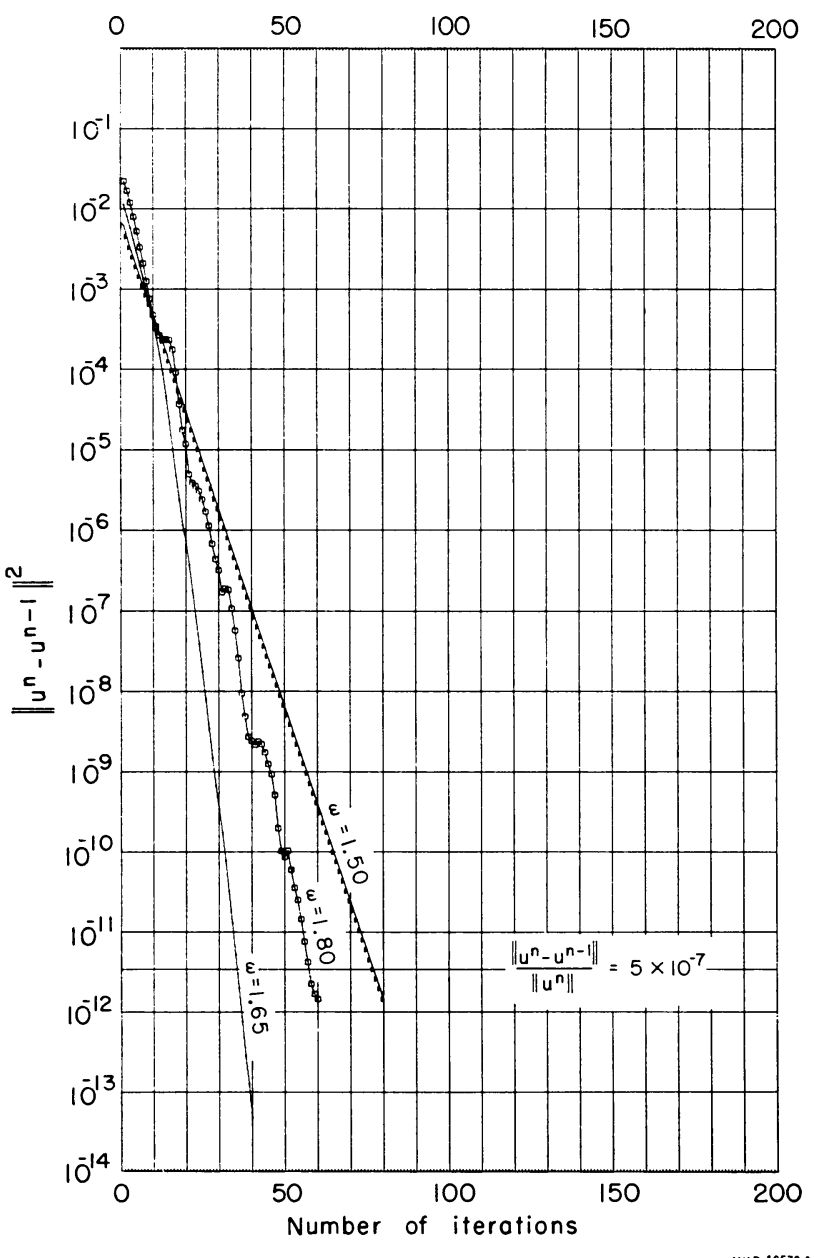

Figure 2. Iteration-by-iteration behavior of ||$u^{n}-u^{n-1}||$ for $K=1, h=1 / 10$, and the harmonic initial approximation. 
effect on the solution of increasing $K$ is depicted in Fig. 1. The contour lines are drawn at intervals of $K / 10$, and, in each case, the maximum height, $K$, occurs in the lower right corner. The surface in the upper left is the limiting solution for the case in which $K \ll 1$,

$$
u=K \frac{\sin (\pi x / 2) \sinh \pi(1-y) / 2}{\sinh (\pi / 2)},
$$

which is the solution of Laplace's equation for the given boundary conditions. The other three surfaces are those obtained numerically for a mesh with $h=1 / 40$. The $K=1 / 2$ and 1 cases are of the degree of nonlinearity one finds in most magnetostatic problems. The $K=5$ case is more nonlinear; the mesh size used yields a sizeable truncation error for it, and the depicted solution of the finite-difference equations is not necessarily a precise representation of the actual minimal surface. The discontinuous slopes of the contour lines for this case result from the piecewise linear interpolation of the contour-plotting program. Gaps in the contour lines are due to the occasional skipping of the pen in the automatic plotting mechanism.

In the numerical tests, three initial approximations are used for the unknown values of $u$ (all initial approximates satisfy the boundary conditions). They are $u=0, u=K$, and the harmonic function, Eq. (12). A few comparisons were made between calculating $\gamma$ from Eq. (4), by use of the machine square-root subroutine (on the IBM 7044), and calculating $\gamma$ approximately from Eq. (10). The comparison problems took the same number of iterations to converge, but in the latter case required $22 \%$ less computer time. Consequently, all test problems reported here use Eq. (10) to calculate $\gamma$, and no other attempt was made to use the exact formula, Eq. (4). Most of the test problems were run by using a Chippewa FORTRAN IV Program on the CDC 6600 Computer, and some were run by using the same program, but in FORTRAN IV, on the IBM 7044. The typical time required was about 0.00028 second per point per iteration [one computation of Eq. (9), using Eqs. (10) and (11), including the necessary peripheral manipulations] on the 6600 .

Table I gives the general numerical results and information concerning the optimal convergence. All quantities are accurate at least to one place in the last digit given. The column for $K \rightarrow 0$ lists the limiting theoretical quantities for small $K$. The other columns list the observed quantities obtained from the test problems. The condition number, area (of the half-problem of the unit square), and angle between the minimal surface and the vertical plane at $x=1, y=0$ are obtained by extrapolation to $h=0$ from the solutions for the different values of $h$. The initial approximation used for each value of $K$ is that one of the three considered that is closest to the solution.

For each of the three mesh sizes, the first row gives the minimal number of iterations required to reduce the relative error $\left\|u^{n}-u^{n-1}|| /|| u^{n}\right\|$, in the Euclidean norm, to less than $5 \times 10^{-7}$. The minimal number of iterations is achieved for the relaxation parameter, $\omega_{\mathrm{opt}}$, given in the second row. The values of $\omega_{\mathrm{opt}}$ were determined empirically to the nearest 0.01 . The quantities $\omega_{b}$ given in the third row are the optimal relaxation parameters (to the nearest 0.01 ) that one would obtain by using the formula applicable to point-successive overrelaxation for linear problems with a coefficient matrix having property (A) [3, §22.1], 


$$
\omega_{b}=2 /\left[1+\left(1-\lambda^{2}\right)^{1 / 2}\right]
$$

where

$$
\lambda=(\eta+\omega-1) / \omega \eta^{1 / 2} .
$$

The spectral radius $\eta$ is estimated by taking the ratio $\left\|u^{n}-u^{n-1}\right\| /\left\|u^{n-1}-u^{n-2}\right\|$, once the ratio asymptotically becomes reasonably constant for an $\omega$ slightly less than $\omega_{\text {op t }}$. The important observation to make is that the value of $\omega_{b}$ thus estimated is essentially the same as the empirically observed value of $\omega_{\text {opt }}$, even though the Jacobian matrix does not have property (A). Also, it should be noted that for the same mesh spacing the values of $\omega_{b}$ and $\eta$ increase as $K$ increases and as the problem becomes more nonlinear.

The observation that the empirically best $\omega_{\text {opt }}$ for fastest global convergence is essentially the same as the asymptotic value of $\omega_{b}$ allows one to closely estimate $\omega_{\text {opt }}$, as the iteration progresses, in the same way as he would for a linear problem possessing property (A). This procedure was utilized successfully by Winslow in solving the magnetostatic equation with a triangular mesh [4]. Another relationship that may be profitably used is the one for small $h$,

$$
\omega_{b} \approx 2 /(1+\alpha h) \quad(h \ll 1) .
$$

This equation was utilized in the test problems to estimate $\omega_{b}$ for a smaller value of $h$ from the value of $\alpha$ that was obtained for the same problem with a larger value of $h$.

Table II shows the results of running the test problem with $K=1, h=1 / 10$, and the harmonic initial approximation for a range of values of $\omega$. These illustrate that the value of $\omega_{b}$ one would estimate does not change greatly for the different values of $\omega<\omega_{b}$. Of course, if the Jacobian matrix had property (A), then the estimates should all be the same. The qualitative behavior is similar to that of a linear problem possessing property (A). That is, the dominant eigenvalue, $\eta$, of the iteration matrix is real and decreases with $\omega$ for $\omega$ between 0 and approximately $\omega_{b}$, rather sharply near $\omega_{b}$. This dominant eigenvalue becomes complex for larger values of $\omega$, and the convergence rate decreases less steeply with $\omega$ as $\omega$ increases from its optimal value than when it decreases from it. This behavior is depicted in Fig. 2, where the iteration-by-iteration behavior for the test problems with $\omega=$ 1.5, 1.65 (optimal), and 1.8 described in Table II are shown. The square of $\left\|u^{n}-u^{n-1}\right\|$ is plotted on a logarithmic scale vs the number of iterations.

In Table III, the maximal values of $\omega$ for which the iteration is convergent for the different initial approximations for $h=1 / 10$ and for $K=1$ and 5 are given. Schechter has shown that to insure convergence for any initial guess, one should not allow $\omega$ to be too large [6]. For example, his results yield that the iteration can be guaranteed to converge for this problem if one chooses $\omega$ less than approximately twice the reciprocal of the condition number, which, for most of the test problems, would be a number considerably less than unity (see Table I). However, from Table III, one sees that for the considered initial approximations, although it is necessary to use a value of $\omega$ less than the optimal to obtain convergence for the poorer approximations, it is possible to use a much larger value of $\omega$ than that required in [6]. Since the convergence rate is considerably lowered by using too small an $\omega$, it would seem best to proceed as follows for reasonable first approximations: Initially, 
TABLE I. Comparison of first test problems

\begin{tabular}{|c|c|c|c|c|c|}
\hline & $\begin{array}{l}K \\
\text { condition no. } \\
A \text { (half area) } \\
\text { Angle with vertical } \\
\text { at }(1,0) \\
\text { initial approx. }\end{array}$ & $\begin{array}{c}\vec{\rightarrow} 0 \\
1 \\
(90-98.13 K)^{\circ}\end{array}$ & $\begin{array}{c}1 / 2 \\
2.0 \\
1.099 \\
44^{\circ} \\
\text { harmon }\end{array}$ & $\begin{array}{c}1 \\
14 \\
1.332 \\
16^{\circ} \\
\text { harmonic }\end{array}$ & $\begin{array}{c}5 \\
>10^{4} \\
3.801 \\
<0.2^{\circ} \\
0\end{array}$ \\
\hline $\begin{array}{l}h=1 / 10 \\
90 \text { pts. }\end{array}$ & $\left\{\begin{array}{l}\text { iterations to converge } \\
\omega_{\text {opt }} \\
\omega_{b}\end{array}\right.$ & 1.606 & $\begin{array}{r}26 \\
1.63 \\
1.62\end{array}$ & $\begin{array}{l}36 \\
1.65 \\
1.65\end{array}$ & $\begin{array}{l}54 \\
1.72 \\
1.72\end{array}$ \\
\hline $\begin{array}{l}h=1 / 20 \\
380 \text { pts. }\end{array}$ & $\left\{\begin{array}{l}\text { iterations to converge } \\
\omega_{\mathrm{opt}} \\
\omega_{b}\end{array}\right.$ & 1.780 & $\begin{array}{l}49 \\
1.80 \\
1.79\end{array}$ & $\begin{array}{l}65 \\
1.81 \\
1.81\end{array}$ & $\begin{array}{l}105 \\
\quad 1.84 \\
1.84\end{array}$ \\
\hline $\begin{array}{l}h=1 / 40 \\
1560 \text { pts. }\end{array}$ & $\left\{\begin{array}{l}\text { iterations to converge } \\
\omega_{\text {opt }} \\
\omega_{b}\end{array}\right.$ & 1.883 & $\begin{array}{r}101 \\
1.89 \\
1.89\end{array}$ & $\begin{array}{r}126 \\
1.90 \\
1.90\end{array}$ & $\begin{array}{l}242^{*} \\
\quad 1.92 \\
1.92\end{array}$ \\
\hline
\end{tabular}

* First 20 iterations at $\omega=1.8$, and next 20 at $\omega=1.9$ to prevent divergence.

TABLE II. Iteration behavior as a function of $\omega$ for $K=1, h=1 / 10$, and the harmonic initial approximation

\begin{tabular}{c|c|c|c}
\hline$\omega$ & $\begin{array}{c}\text { iterations } \\
\text { to converge }\end{array}$ & $\eta$ & $\omega_{b}$ \\
\hline 1.0 & 227 & 0.959143 & 1.6637 \\
1.2 & 156 & 0.938429 & 1.6634 \\
1.4 & 101 & 0.901931 & 1.6627 \\
1.5 & 78 & 0.869023 & 1.6616 \\
1.6 & 63 & 0.80318 & 1.6584 \\
1.63 & 45 & 0.758 & 1.655 \\
1.65 & 36 & $*$ & (optimal) \\
1.67 & 37 & $*$ & \\
1.7 & 40 & $*$ & \\
1.8 & 58 & $*$ & \\
1.9 & 124 & & \\
\hline
\end{tabular}

* Oscillatory

TABLE III. Maximal $\omega$ for which iteration will converge for $h=1 / 10$

\begin{tabular}{|c|c|c|c|}
\hline$K$ & $\omega_{o p t}$ from Table I & initial approx. & $\omega_{\max }$ \\
\hline 1 & 1.65 & $\begin{array}{c}\text { harmonic } \\
0 \\
1\end{array}$ & $\begin{array}{l}1.97 \\
1.83 \\
1.60\end{array}$ \\
\hline$\tilde{j}$ & 1.72 & $\begin{array}{c}\text { harmonic } \\
0 \\
\tilde{5}\end{array}$ & $\begin{array}{l}1.63 \\
1.85 \\
1.44\end{array}$ \\
\hline
\end{tabular}


try to use a value of $\omega$ equal to the estimated $\omega_{\text {opt }}$. If the iteration then diverges, slightly smaller values of $\omega$ should be tried until one is found for which the iteration does not diverge. Then $\omega$ should be increased toward the optimal as the approximation to the solution improves. Such was the procedure for the $h=1 / 40, K=5$ example in Table I.

4.3. Results for second problem. Tables IV and V compare the discretization error obtained by the present method in solving the second test problem, Eq. (6), with that obtained by Greenspan with his lower-order, one-sided differencing scheme [2]. The problem has the exact solution $u_{\mathrm{E}}=\left(\cosh ^{2} y-x^{2}\right)^{1 / 2}$. An initial approximation was obtained by linear interpolation of the boundary values, as it was in [2] The specific interpolation used here is

$$
u(x, y)=u(x, 0)+y[u(x, 1)-u(x, 0)], \quad(0<x<1,0<y<1) .
$$

For $h=1 / 40$ and $\omega=1.87$, the present method requires 106 iterations to converge to $\left\|u^{n}-u^{n-1}|| /|| u^{n}\right\|<5 \times 10^{-7}$ and 162 iterations to $<10^{-9}$; for $h=1 / 50$ and $\omega=1.89,127$ and 192 iterations. The ordering of the iterations and computer time required are the same as for the first test problem. The actual optimal values of $\omega$ were not determined, but, from the asymptotic convergence rate, the values used

TABLE IV

Comparison of discretization errors with those of Greenspan;

$$
h=1 / 40
$$

\begin{tabular}{|c|c|c|c|c|}
\hline \multirow[b]{2}{*}{$y$} & \multirow[b]{2}{*}{$x$} & \multicolumn{2}{|c|}{$u$} & \multirow[b]{2}{*}{$u_{\mathrm{E}}$} \\
\hline & & This method & $\begin{array}{c}\text { Reference [2] } \\
\text { Table IV }\end{array}$ & \\
\hline 0.00 & 0.95 & 0.31224990 & 0.31224990 & 0.31224990 \\
\hline 0.05 & 0.65 & 0.76157878 & 0.76097338 & 0.76157868 \\
\hline 0.10 & 0.30 & 0.95918333 & 0.95901362 & 0.95918370 \\
\hline 0.10 & 0.80 & 0.60832683 & 0.60584338 & 0.60830369 \\
\hline 0.15 & 0.55 & 0.84863211 & 0.84775599 & 0.84862787 \\
\hline 0.20 & 0.40 & 0.93837174 & 0.93792401 & 0.93836890 \\
\hline 0.20 & 0.95 & 0.37240933 & 0.36438793 & 0.37153221 \\
\hline 0.25 & 0.20 & 1.01183731 & 1.01175927 & 1.01183644 \\
\hline 0.30 & 0.70 & 0.77640646 & 0.77433730 & 0.77635856 \\
\hline 0.40 & 0.55 & 0.93073514 & 0.92996610 & 0.93070805 \\
\hline 0.45 & 0.95 & 0.56063992 & 0.55867880 & 0.56039557 \\
\hline 0.50 & 0.35 & 1.07194587 & 1.07201608 & 1.07193298 \\
\hline 0.65 & 0.65 & 1.03104877 & 1.03106640 & 1.03099812 \\
\hline 0.70 & 0.85 & 0.92363372 & 0.92351784 & 0.92355251 \\
\hline 0.75 & 0.15 & 1.28596932 & 1.28630885 & 1.28596454 \\
\hline 0.75 & 0.50 & 1.19426367 & 1.19475354 & 1.19423817 \\
\hline 0.80 & 0.20 & 1.32240243 & 1.32280683 & 1.32239640 \\
\hline 0.85 & 0.45 & 1.30831865 & 1.30883314 & 1.30830338 \\
\hline 0.85 & 0.95 & 1.00584466 & 1.00600982 & 1.00581197 \\
\hline 0.90 & 0.30 & 1.40133993 & 1.40171238 & 1.40133386 \\
\hline 0.90 & 0.70 & 1.25052009 & 1.25094661 & 1.25049454 \\
\hline 0.95 & 0.05 & 1.485384506 & 1.48545280 & 1.48538405 \\
\hline 0.95 & 0.65 & 1.33656165 & 1.33683577 & 1.33654995 \\
\hline
\end{tabular}




\section{TABLE V}

Comparison of discretization errors with those of Greenspan;

$$
h=1 / 50
$$

\begin{tabular}{|c|c|c|c|c|}
\hline \multirow[b]{2}{*}{$y$} & \multirow[b]{2}{*}{$x$} & \multicolumn{2}{|c|}{$u$} & \multirow[b]{2}{*}{$u_{\mathrm{E}}$} \\
\hline & & This method & $\begin{array}{l}\text { Reference [2] } \\
\text { Table V }\end{array}$ & \\
\hline 0.00 & 0.96 & 0.28000000 & 0.28000000 & 0.28000000 \\
\hline 0.04 & 0.66 & 0.75233010 & 0.75213765 & 0.75233028 \\
\hline 0.10 & 0.30 & 0.95918346 & 0.95912198 & 0.95918370 \\
\hline 0.10 & 0.80 & 0.60831775 & 0.60740805 & 0.60830369 \\
\hline 0.16 & 0.56 & 0.84393414 & 0.84357683 & 0.84393080 \\
\hline 0.20 & 0.40 & 0.93837067 & 0.93820524 & 0.93836890 \\
\hline 0.20 & 0.94 & 0.39659419 & 0.39344046 & 0.39615172 \\
\hline 0.24 & 0.20 & 1.00931433 & 1.00928742 & 1.00931385 \\
\hline 0.30 & 0.70 & 0.77638884 & 0.77558328 & 0.77635856 \\
\hline 0.40 & 0.56 & 0.92474375 & 0.92442376 & 0.92472562 \\
\hline 0.44 & 0.96 & 0.53384849 & 0.53294933 & 0.53368591 \\
\hline 0.50 & 0.36 & 1.06862472 & 1.06864723 & 1.06861608 \\
\hline 0.66 & 0.66 & 1.03300908 & 1.03299358 & 1.03297588 \\
\hline 0.70 & 0.84 & 0.93270829 & 0.93260872 & 0.93265708 \\
\hline 0.74 & 0.14 & 1.27888747 & 1.27901338 & 1.27888461 \\
\hline 0.74 & 0.50 & 1.18540510 & 1.18557830 & 1.18538848 \\
\hline 0.80 & 0.20 & 1.32240025 & 1.32255781 & 1.32239640 \\
\hline 0.84 & 0.94 & 1.00221326 & 1.00225312 & 1.00218885 \\
\hline 0.86 & 0.46 & 1.31503763 & 1.31522812 & 1.31502800 \\
\hline 0.90 & 0.30 & 1.40133772 & 1.40148232 & 1.40133386 \\
\hline 0.90 & 0.70 & 1.25051094 & 1.25066097 & 1.25049454 \\
\hline 0.94 & 0.64 & 1. 32926546 & 1.32938045 & 1. 32925684 \\
\hline 0.96 & 0.06 & 1.49609247 & 1.49611899 & 1.49609203 \\
\hline
\end{tabular}

were estimated to be within, at most, about $1 \%$ of the optimal. The discretization errors were $\left\|u^{162}-u_{\mathrm{E}}\right\| /\left\|u_{\mathrm{E}}\right\|=1.634 \times 10^{-4}$ for $h=1 / 40 ;\left\|u^{192}-u_{\mathrm{E}}|| /|| u_{\mathrm{E}}\right\|$ $=1.244 \times 10^{-4}$ for $h=1 / 50$.

For $h=1 / 40$ and $\omega=1.24,375$ iterations were required in Example 10 of [2] for the same problem to converge (the exact convergence criterion was not given); for $h=1 / 50$ and $\omega=1.6$, divergence occurred [2, Example 13]. The time required per point per iteration there was about $0.001 \mathrm{sec}$ on the CDC 1604. Although a precise comparison is not possible because of the difficulty of comparing different programs on different computers, the higher-order differencing scheme used here apparently may, in addition to lowering the discretization error, have improved the convergence.

5. Summary. The promising results obtained for the test problems suggest that the method discussed here is a useful one for solving the minimal surface equation. In general terms, the important points are (1) setting up the difference equations in the manner suggested, using the central approximation for $|\nabla u|^{2}$, and (2) solving these equations by using nonlinear successive overrelaxation, estimating the optimal re- 
laxation parameter by use of the formulas valid for linear problems with coefficient matrices possessing property (A). If the iteration diverges, then a smaller relaxation parameter should be used initially and adjusted towards the optimal, after first allowing the iteration to proceed a number of steps.

Acknowledgment. The author is indebted to Mrs. Marjory Simmons for obtaining the contour plots of Fig. 1 from the tabular data and to the referees for their helpful comments.

Lawrence Radiation Laboratory

Berkeley, California 94720

1. P. Concus, "Numerical solution of the nonlinear magnetostatic-field equation in two dimensions," J. Comput. Phys., v. 1, No. 3, 1966-1967. (To appear.)

2. D. Greenspan, "On approximating extremals of functionals. I. The method and examples for boundary value problems," ICC Bull., v. 4, 1965, pp. 99-120. MR 32 \#526.

3. G. E. Forsythe \& W. R. WAsow, Finite-Difference Methods for Partial Differential Equations, Wiley, New York, 1960. MR 23 \#B3156.

4. A. M. WINsLOw, "Numerical solution of the quasi-linear Poisson equation in a nonuniform triangle mesh," J. Comput. Phys., v. 1, no. 2, 1966-1967.

5. J. M. OrTega \& M. L. RockofF, "Nonlinear difference equations and Gauss-Seidel type iterative methods," SIAM J. Numer. Anal., v. 3, 1966, pp. 497-513.

6. S. Schechter, "Iteration methods for nonlinear problems," Trans. Amer. Math. Soc., v 104, 1962, pp. 179-189. MR $27 \# 2122$. 